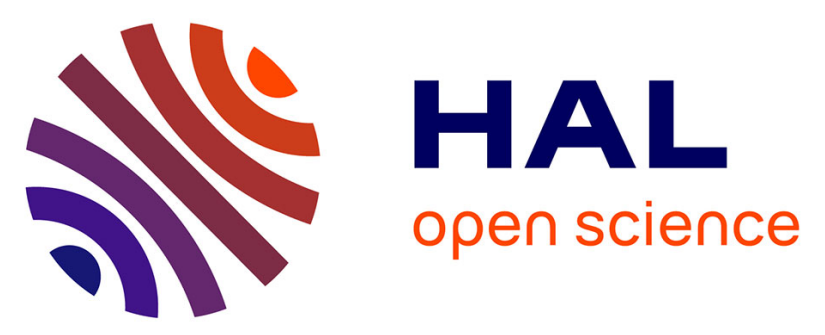

\title{
Rituximab is an effective and safe therapeutic alternative in adults with refractory and severe autoimmune hemolytic anemia
}

Francisco Javier Peñalver, Alberto Alvarez-Larrán, Jose Luis Díez-Martin, Laura Gallur, Isidro Jarque, Dolores Caballero, Joaquín Díaz-Mediavilla, Rosalía Bustelos, María Jesús Fernández-Aceñero, José Rafael Cabrera

\section{To cite this version:}

Francisco Javier Peñalver, Alberto Alvarez-Larrán, Jose Luis Díez-Martin, Laura Gallur, Isidro Jarque, et al.. Rituximab is an effective and safe therapeutic alternative in adults with refractory and severe autoimmune hemolytic anemia. Annals of Hematology, 2010, 89 (11), pp.1073-1080. 10.1007/s00277010-0997-y · hal-00543002

\section{HAL Id: hal-00543002 https://hal.science/hal-00543002}

Submitted on 5 Dec 2010

HAL is a multi-disciplinary open access archive for the deposit and dissemination of scientific research documents, whether they are published or not. The documents may come from teaching and research institutions in France or abroad, or from public or private research centers.
L'archive ouverte pluridisciplinaire HAL, est destinée au dépôt et à la diffusion de documents scientifiques de niveau recherche, publiés ou non, émanant des établissements d'enseignement et de recherche français ou étrangers, des laboratoires publics ou privés. 
Editorial Manager(tm) for Annals of Hematology

Manuscript Draft

Manuscript Number: AOHE-D-09-00144R2

Title: RITUXIMAB IS AN EFFECTIVE AND SAFE THERAPEUTIC ALTERNATIVE IN ADULTS WITH REFRACTORY AND SEVERE AUTOIMMUNE HEMOLYTIC ANEMIA

Article Type: Original Article

Keywords: Autoimmune hemolytic anemia; Refractory AIHA; Rituximab; Hematological response

Corresponding Author: Dr Francisco Javier Peñalver,

Corresponding Author's Institution:

First Author: Francisco Javier Peñalver

Order of Authors: Francisco Javier Peñalver; Alberto Álvarez-Larrán; Jose Luis Díez-Martín; Laura Gallur; Isidro Jarque; Dolores Caballero; Joaquín Díaz-Mediavilla; Rosalía Bustelos; María Jesús Fernández-Aceñero; José Rafael Cabrera

Abstract: Rituximab-induced B cell depletion has been proven to be a useful therapy for autoimmune hemolytic anemia (AIHA). The aim of this retrospective study was to evaluate the effectiveness of rituximab in the treatment of 36 patients with AIHA refractory to several treatments. These patients had received a median of four (1-8) previous treatments, and 13 patients had undergone splenectomy. Rituximab was administered by intravenous infusion at a dose of $375 \mathrm{mg} / \mathrm{m} 2$ once weekly for 4 doses in 29 patients, and 7 patients received 1-6 doses. Overall, 28 of 36 patients (77\%) achieved response. Twenty-two patients (61\%) reached a complete response (CR), and 6 patients (16\%) obtained a partial response (PR). All patients that reached CR (61\%) were able to maintain the response during more than 6 months with a median follow-up of 14 months (1-86 months). Sixteen patients maintained response (Maintained Response; MR) for more than 1 year. The predictors of MR were achievement of $\mathrm{CR}$ and negative Coombs test result. Splenectomized patients showed a better response rate than those non-splenectomized. Rituximab was well tolerated, and only one patient presented a transitory rash during infusion. Rituximab induced clinical responses in multi-treated severe refractory both warm and cold AIHA patients with little toxicity and consequently, this therapy should be considered as an early therapeutic option in this setting.

Response to Reviewers: In order to read the response to reviewers see attachment. 


\section{RITUXIMAB IS AN EFFECTIVE AND SAFE THERAPEUTIC ALTERNATIVE IN ADULTS WITH REFRACTORY AND SEVERE AUTOIMMUNE HEMOLYTIC ANEMIA}

Francisco Javier Peñalver ${ }^{1}$, Alberto Alvarez-Larrán², Jose Luis Díez-Martin ${ }^{3}$, Laura Gallur $^{4}$, Isidro Jarque ${ }^{5}$, Dolores Caballero ${ }^{6}$, Joaquín Díaz-Mediavilla ${ }^{7}$, Rosalía Bustelos ${ }^{8}$, María Jesús Fernández-Aceñero ${ }^{9}$ and José Rafael Cabrera $^{10}$ on behalf of the Multi-institutional Retrospective Study on the use of rituximab in refractory AIHA

${ }^{1}$ Hospital Universitario Fundación Hospital de Alcorcón, Madrid, Spain; ${ }^{2}$ Hospital Clinic, Barcelona, Spain; ${ }^{3}$ Hospital Gregorio Marañon, Madrid, Spain; ${ }^{4}$ Hospital Vall D’Hebron, Barcelona, Spain; ${ }^{5}$ Hospital La Fe, Valencia, Spain; ${ }^{6}$ Hospital Clínico de Salamanca, Salamanca, Spain; ${ }^{7}$ Hospital Clínico de Madrid, Madrid, Spain; ${ }^{8}$ Hospital Universitario 12 de Octubre, Madrid, Spain; ${ }^{9}$ Fundación Jiménez Díaz, Biostatistics, Madrid, Spain; ${ }^{10}$ Hospital Universitario Puerta de Hierro, Madrid, Spain.

\section{Corresponding author}

Dr. Francisco Javier Peñalver Hospital Universitario Fundación Alcorcón

C/Budapest, 1. 28922. Alcorcón. Madrid. Spain.

Tel: 34-916219494

Fax: 34-916219901

E-mail: fjpenalver@fhalcorcon.es 
Abstract

Rituximab-induced B cell depletion has been proven to be a useful therapy for autoimmune hemolytic anemia (AIHA). The aim of this retrospective study was to evaluate the effectiveness of rituximab in the treatment of 36 patients with AlHA refractory to several treatments. These patients had received a median of four (1-8) previous treatments, and 13 patients had undergone splenectomy. Rituximab was administered by intravenous infusion at a dose of $375 \mathrm{mg} / \mathrm{m}^{2}$ once weekly for 4 doses in 29 patients, and 7 patients received 1-6 doses. Overall, 28 of 36 patients (77\%) achieved response. Twenty-two patients $(61 \%)$ reached a complete response $(\mathrm{CR})$, and 6 patients (16\%) obtained a partial response $(\mathrm{PR})$. All patients that reached CR (61\%) were able to maintain the response during more than 6 months with a median follow-up of 14 months (186 months). Sixteen patients maintained response (Maintained Response; MR) for more than 1 year. The predictors of MR were achievement of $C R$ and negative Coombs test result. Splenectomized patients showed a better response rate than those non-splenectomized. Rituximab was well tolerated, and only one patient presented a transitory rash during infusion. Rituximab induced clinical responses in multi-treated severe refractory both warm and cold AlHA patients with little toxicity and consequently, this therapy should be considered as an early therapeutic option in this setting. 
Introduction

Autoimmune haemolytic anemia (AlHA) is a disorder in wich $\lg$ and/or $\lg$ M autoantibodies are directed against autologous red blood cells (RBC) surface antigens and iniciate $\mathrm{RBC}$ destruction by the reticuloendothelial system and complement system, respectively. About $80 \%$ of patients which suffer from AlHA have warm-reactive antibodies of the $\lg G$ isotype; the remainder exibit cold-reactive autoantibodies. Although AlHA can be idiopathic, $20-80 \%$ of patients have a suspected secondary cause such as lymphoproliferative disorders (LD), autoimmune disorders (AD) and infections ${ }^{1}$.

In patients with warm AlHA (WAIHA) requiring treatment, corticosteroids represent the first-line therapy, even though only $15-20 \%$ of new cases achieve a complete remision ${ }^{1}$. For those patients initially unresponsive to corticosteroids, the second-line of therapy is usually splenectomy, which is associated with an initial response rate of aproximately $50 \%{ }^{2}$. Treatment options for patients who fail to respond, relapse or are unable to undergo splenectomy are limited. Citotoxic and immunosuppresant drugs, such as cyclophosphamide, azathioprine and ciclosporine A, provide a 40-60\% response rate. However, these treatments may be associated with serious side effects. The effectiveness of other options, such us intravenous immunoglobulin (IVIG), plasmapheresis and danazol, is controversial ${ }^{1,2,3}$.

In patients with cold AIHA (CAIHA), therapy based on corticosteroids is generally not helpful and splenectomy is not usually effective, as complementcoated RBC is mainly destroyed in the liver ${ }^{4}$. In cases with severe hemolysis, immunosuppression with chlorambucil or cyclophosphamide may be beneficial 
and plasmapheresis can provide transitory improvement. Nevertheless, this therapy is generally unsatisfactory ${ }^{1,2,4}$.

The aetiology of autoantibody production is not well understood, but is thought to involve a complex dysfuntion of the immune system and immune surveillance mechanism ${ }^{5}$. Despite the complex pathogenic mechanism of AIHA, B cells play a crucial role in the pathogenesis of these disorders ${ }^{6}$.

Rituximab is a humanized chimeric anti-CD20 monoclonal antibody specific for the CD20 antigen expressed on all normal and malignant mature Blymphocytes. After introduction of rituximab as a powerful therapeutic agent in B-cell non-Hodgkin's Lymphoma, numerous attemps have been made to use Blymphocyte depletion induced by rituximab for the treatment of both nonhaematological and haematological autoimmune diseases ${ }^{4,6,7,8}$.

Similar to other autoimmune cytopenias, AlHA is a rare disease, which estimated incidence is $1-3$ cases per 100.000 per year $^{1}$, making it difficult to perform randomized trials. Up to now, non-randomized prospective studies are relatively few, and restrospective series of consecutive patients are often small ${ }^{9}$. An overview of studies including five or more patients reveals that rituximab is effective in treating warm and cold AlHA, with an overall response rate ranging from 40 to $100 \%$ (median $60 \%$ ), and with patients of all ages responding ${ }^{4}$. Therefore, controlled studies that include larger numbers of patients that have received severals treatments in different clinical situations (associated diseases, splenectomy or not) are necessary to asses the role of rituximab in the treatment of adult patients with refractory and severe AlHA. 
In the present study, we show the results of a retrospective multicentric Spanish study designed to asses the therapeutic efficacy and toxicity of rituximab in refractory and severe AlHA patients unresponsive to several treatments. 


\section{Design and methods}

Our group has performed a restrospective multicentric analysis of adult patients with AlHA treated with rituximab between April 2001 and June 2007 in 21 centres in Spain.

Inclusion criteria

In the present study, we have included adult patients with AlHA, both idiopathic AlHA and associated with LD and AD.

Diagnosis of AlHA was based on laboratory evidence of hemolysis (increased reticulocyte count with increased indirect bilirrubin, lactate dehydrogenase, or absent haptoglobin levels) and a positive Coombs test.

Refractory and severe AlHA patients were elegible for treatment with rituximab. We defined as refractory AlHA those patients with hemoglobin levels $<11 \mathrm{~g} / \mathrm{dL}$ prior to rituximab treatment who had received at least two therapy lines and patients with hemoglobin levels $>11 \mathrm{~g} / \mathrm{dL}$ prior to rituximab who had received at least three therapy lines and with treatment dependence to maintain this level of hemoglobin before rituximab initiation. All patients were negative for hepatitis $B$ virus $(\mathrm{HBV})$ and hepatitis $\mathrm{C}$ virus $(\mathrm{HCV})$. All patients gave informed consent to receive the rituximab under off-label conditions. This study was approved by the local ethics committee, and informed consent for analysing patients' data was obtained.

\section{$\underline{\text { Treatment }}$}

Patients received a weekly dose of $375 \mathrm{mg} / \mathrm{m}^{2}$ rituximab (Mabthera; Roche Farma, Spain) intravenously for 4 consecutive weeks. During the initial period, 26 multitransfused patients with severe anemia and/or requiring a maintenance dose of therapy received rituximab associated with other therapies as follows: 
corticosteroids (9 of 26 patients), corticosteroids and cyclosphosphamide (6 of 26 patients), corticosteroids and other treatments they were previously receiving (8 of 26 patients) and other treatments (2 of 26 patients).

\section{$\underline{\text { Response criteria }}$}

The response criteria were defined as follows: complete response (CR), nontransfused hemoglobin level $>12 \mathrm{~g} / \mathrm{dL}$ and hemoglobin increase of at least $>2 \mathrm{~g} / \mathrm{dL}$ above their pretreatment hemoglobin level with the discontinuation of concomitant immunosuppressive therapies; partial response (PR), nontransfused hemoglobin level $>10 \mathrm{~g} / \mathrm{dL}$ and hemoglobin increase of at least 2 $\mathrm{g} / \mathrm{dL}$ above their pretreatment hemoglobin levels. Maintained response (MR) was defined as the maintenance of response for more than 6 months after the beginning of treatment and with the discontinuation of concomitant immunosuppressive therapy.

Statistical analysis

Analysis of the data was primarily descriptive, using percentages, mean values and standard deviations, medians and ranges as required. Chi-squared test was used to compare proportions and Student's $t$ test to compare means. We have also represented the results with graph bars including means and 95\% confidence intervals $(\mathrm{Cl})$ and box plots with percentiles and extreme values.

A Kaplan-Meier analysis was performed to represent probability of maintaining response in patients and in order to estimate the median and mean of MR. The event considerate to perform this analysis was losing MR and we censured those patients that had reached the endpoint of maintenance of response. We use the Kaplan-Meier program contained in SPSS ver 13.0. 


\section{Results}

We analyzed 36 adult patients with AlHA, both idiopathic AIHA and associated with LD and AD.

\section{$\underline{\text { Pretreatment characteristics }}$}

Patients' demographics and clinical characteristics are summarized in Table 1. Prior to rituximab, 31 patients had received three or more treatments, with a median of 4 (range 1-8). The previous treatment included corticosteroids (97\%); cyclophosphamide (47\%); IVIG (42\%); cyclosporine (28\%), azathioprine (14\%) and chlorambucil (11\%).

Splenectomized patients had received a median of 5 treatments before rituximab. $77.8 \%$ of the patients (28 out of 36 ) had been transfused.

We have also included 5 multitransfused patients with severe AlHA, that showed hemoglobin levels $<8 \mathrm{~g} / \mathrm{dL}$ (median hemoglobin levels of $6 \mathrm{~g} / \mathrm{dL}$, range 4-8 $\mathrm{g} / \mathrm{dL}$ ), prior to rituximab, and who had received one therapy line, either corticosteroids (including high dose, 4 patients) or cyclosporine before receiving rituximab.

Overall, $80.5 \%$ (29 out of 36 ) of patients received four doses of rituximab, and $19.5 \%$ ( 7 of 36 ) of patients received between one and six doses of rituximab (one patient, one dose, five doses and six doses each; two patients, two doses and three doses each).

\section{$\underline{\text { Response to treatment and its characteristics }}$}

The overall response rate for patients receiving rituximab was $77 \%$. Twenty-two patients (61\%) achieved CR and 6 patients (16\%) obtained PR (Figure 1). 
A large proportion of responses took place between the first and third week of treatment ( $82 \%$ of the responders, 18 patients). The median time to have a response was 3 weeks (range 1-8 weeks). We also found late responses in five patients between 6-8 weeks (Figure 2).

Maximum response was achieved after a median time of 10 weeks (range 4-35 weeks), with median hemoglobin levels of $13 \mathrm{~g} / \mathrm{dL}$ (range $10-15.6 \mathrm{~g} / \mathrm{dL}$ ) The increase of hemoglobin levels since pre-treatment conditions was statistically significant $(p<0.001)$ (Figure 3).

All patients with PR showed an increase of hemoglobin $>3 \mathrm{~g} / \mathrm{dL}$ above their pre-treatment hemoglobin levels, with a median increase of hemoglobin posttreatment of $5 \mathrm{~g} / \mathrm{dL}$ (range $3-7.5 \mathrm{~g} / \mathrm{dL}$ ), a median hemoglobin post-treatment of $10.8 \mathrm{~g} / \mathrm{dL}$ (range 10-11.7 $\mathrm{g} / \mathrm{dL}$ ) and a median duration of response of 4 months (range 1-9 months).

$\underline{\text { Response maintenance }}$

Overall, response was maintained in all the patients that had reached CR (61 $\%, 22$ out of 36 ), with a median duration of response of 19 months (range 6-86 months), and 16 patients were able to maintain the response even more than a year with a median duration of 23 months (Figure 1).

In Kaplan-Meier analysis, the estimated mean of MR over the study period was 65.9 months (95\% confidence interval [CI]: 52.9-78.9). The estimated probability of maintaining response between 6 and 83 months was estimated around $75 \%$. At the end of the study period, median of MR was not achieved. We censured those patients that had maintained response. It must be taking in account that the estimated mean of MR obtained by Kaplan-Meier must be assumed with 
caution as patients who had maintained their response for the longest periods of time were censured (Figure 4).

\section{Factors associated with response}

There were no differences in the response rate between patients with warm (CR and MR $61.5 \% ; 16$ out of 26 ) or cold AIHA (CR and MR $66.7 \% ; 6$ out of 9 ) $(p=0.9)$. Moreover, the duration of MR was not significantly different between warm AlHA patients (median 15 months, range 6-86 months) and cold AlHA patients (median 21 months, range 17-36 months) ( $p=0.62)$.

We did not find noteworthy statistical differences in CR and MR rates and duration of response between patients with idiopathic AlHA or those with AIHA associated to LD, AD, allogenic stem cell and liver transplant. Fifty percent (7 out of 14) of patients with idiopathic AlHA and 69\% (11 out of 16) of patients with AlHA associated to LD experienced CR. We found non-significant differences between both groups in $C R$ and $M R(p=0.5)$ and duration of response (21 and 14 months; idiopathic disease and AIHA associated to LD respectively; $p=0.73$ ). Two out of 4 patients with cold AIHA associated to LD reached $C R$ and all of them were to able maintained it with a median duration of response of 19 and 29 months respectively.

Most of patients that had maintained response reached a negative result from Coombs test (83.3\%; 15 out of 18 ). The frequency of patients that achieved a MR and showed a negative Coomb test was significantly higher than the percentage of patients with a MR but whose result from Coombs test was positive $(p=0.017)$. On the other hand, we did not found significant differences when we compared the duration of response between these subpopulations of 
patients (15 and 28 months, negative and positive Coomb test respectively; $p=0.65)$ (Figure 5).

Splenectomized patients with warm AlHA showed a trend to an enhanced response rate comparing to non-splenectomized patients, but this difference was not statistically significant $(p=0.3)$. Seventy-five percent of splenectomized patients (9 out of 13) achieved a CR that was maintained for a median of 21 months and $54 \%$ (13 out of 24 ) of non-splenectomized patients showed a CR that was maintained during a median of 19 months. No differences were found in the length of $\mathrm{MR}$ regarding splenectomy $(p=0.67)$. Moreover, we did not found remarkable differences either in CR or MR when we analyzed the responses to rituximab in splenectomized or non splenectomized patients with cold AIHA. Nevertheless, it is worth noting that all splenectomized patients with cold AlHA treated with rituximab achieved CR in relation to less than a half of non-splenectomized (100\% and 50\%; splenectomized and non-splenectomized patients respectively; $p=0.53)$. Furthermore, no differences were found in duration of MR between these groups.

Rituximab therapy alone achieved a CR rate similar to that showed by rituximab in combination with other therapies $(60 \%$ and $58 \%$; monotherapy and combined therapy respectively; $p=0.8)$. Moreover, duration of MR between this subsets of patients was comparable (18 and 20 months respectively; $p=0.6$ ). Six patients with warm AlHA received rituximab in combination with cyclophosphamide and steroids. Five of these patients suffered from AlHA associated LD and they had also received corticosteroids. Four of these patients (80\%) achieved CR and were able to maintain this response with a median of duration of 13 months (range 6-19 months). 
Heavily treated patients before rituximab therapy (more than four treatments, including any corticosteroid) did not present significant differences in terms of response rate than those receiving fewer treatments (CR and MR 54.5\% and $64.3 \% ; p=0.81)$. Similarly, both groups maintained the response during a median of 19 months.

The median time from diagnosis of AlHA to rituximab treatment was 8.7 months. Our findings showed no significant differences in the CR and the maintenance of response between the patients with longer duration of AlHA (> 8 months) and patients with AIHA lasting less than 8 months before rituximab therapy $(55.6 \%$ and $61 \%$ respectively; $p=1)$. The median duration of response between these groups did not showed notable differences (20 and 15.5 months respectively; $p=0.7)$.

Age and gender did not seem to influence response to rituximab therapy (Data not shown).

\section{Toxicity, side effects and infectious complications}

The treatment was generally well tolerated. All patients were pretreated with acetaminophen, diphenhydramine and intravenous hydrocortisone. Only one patient presented a transitory rash during infusion. No infectious complications were comunicated. 


\section{Discussion}

This study aimed to describe the characteristics of response to rituximab in 36 patients with refractory AIHA both idiopathic and associated with AD or LD. Up to now, rituximab had been shown to be effective for the treatment of refractory AlHA in children and in a small number of adults ${ }^{10,11}$. Our findings show that rituximab induces a clinically significant response in patients with AlHA who are unresponsive to other therapeutic options. Overall, response rate for patients receiving rituximab was $77 \%$. Sixty-one percent of patients achieved CR and all of them were able to maintain it. Moreover, this response is not influenced either by gender or age of the patient or by hemoglobin levels prior to treatment in contrast to other reports ${ }^{12,13}$.

To our knowledge, this study includes the largest series reported to date about the use of rituximab in adults with severe refractory $\mathrm{AlHA}^{4}$. Similar findings were obtained in a recent retrospective study including 27 patients with idiopathic or secondary warm $\mathrm{AlHA}{ }^{14}$, in which rituximab led to an initial response in 25 (93\%) cases, with a CR in $8(30 \%)$ and PR in 17 (63\%) patients. Three patients were retreated with rituximab, and the same pattern of response (1CR, 2PR) was obtained. In our series, only one patient was retreated after maintaining the response during 11 months. This patient reached a new CR with 2 doses of rituximab, which was sustained with rituximab every 3 months.

The therapeutic objective of rituximab therapy in refractory AlHA must be achieve CR, and furthermore the CR could be consider as a predictor of MR seeing that all the patients with a CR were able to maintain their response. Although to date, no laboratory parameters predictive of response have been identified ${ }^{15,16}$, our results show that a negative result from Coombs test after 
rituximab administration could be a factor associated to the maintenance of response. The negative result from Coombs test after treatment seem to be an adequate laboratory predictor of maintenance of response seeing that $83.3 \%$ of patients that had achieved CR showed a negative result from Coombs test and they were able to maintain the response for a median of 15 months.

Regarding splenectomy, both splenectomized and non-splenectomized patients have obtained a remarkable response, with a trend to a higher response rate in splenectomized patients with warm AlHA. It has been previously reported that about $60 \%$ of patients who undergo splenectomy achieve a complete remission ${ }^{3,17,18}$. Patients with cold AlHA behaved equally in terms of response whether or not they had been previously splenectomized. Besides, it is worth noting that all splenectomized patients with cold AlHA treated with rituximab achieved CR in relation to less than a half of those non-splenectomized that were able to experience a CR. However, splenectomy did not show an effect on response maintenance either in warm or cold AlHA patients in our study. These findings could support the hypothesis that an intact spleen is not necessary to achieve a response when patients are treated with rituximab in contrast to other studies particularly in ITP ${ }^{19}$. Moreover, previous splenectomy in AlHA patients could favour the response to immunosuppressant agents. In fact, rituximab has demonstrated to be more effective in patients with AlHA refractory to splenectomy, providing a therapeutic alternative to the prolonged administration of immunosuppressant agents.

The aetiology of autoantibodies of AlHA did not influence response to treatment seeing that there were no statistical differences in response rate and duration of response between patients with warm and cold AlHA. Although MR rates 
obtained in treatment of cold AIHA have been generally lower than those reported for the management of warm-antibody disease ${ }^{20}$, the present study shows that rituximab could be effective in obtaining MR in patients with cold AlHA, offering an additional treatment choice to this group with limited options. The present study also includes cases of refractory AlHA secondary to a range of conditions including AD, LD, allogenic stem cell and liver transplant, which have been excluded in other reports ${ }^{21,12}$. We did not find statistically significant differences in CR, MR rate and duration of response between these patients and those with idiopathic refractory AlHA. This data suggests that rituximab could behave as an effective immunosupressor in refractory AlHA despite the cause of this autoantibody mediated disease as it can be seen in other analyses ${ }^{22,23}$.

Rituximab induced rapid responses, most of which took place between the first and third week of infusion. The early response obtained could be attributed to rituximab since a temporal relationship between rituximab infusion and response has been noted. Moreover, these responses seemed to be unaffected by the fact that $86 \%$ of patients received other drugs together with rituximab, mainly corticosteroids, therefore suggesting that rituximab could be the main factor responsible for the therapeutic benefit. Reported therapeutic mechanisms of rituximab include antibody-dependent cell-mediated cytotoxicity (ADCC), complement-dependent cytotoxicity (CDC), and direct apotosis signalling that alter B cell homeostasis and disturb T-cell activation ${ }^{24,25}$. Although the mechanism of action of rituximab may be related to depletion of auto-reactive $B$ cells, early responses in our study could suggest a saturation of Fc receptors of the monocyte-macrophage system by opsonized B cells as an additional 
mechanism preventing the inmmune-mediated destruction of the autoantibody coated erythrocyte as previously described ${ }^{13}$.

We have found that the response rate in patients treated with rituximab in combination with other therapies was not different from that shown in patients with rituximab therapy alone. However, in patients with underlying lymphoproliferative disorders in particular, treatment with a combination of rituximab and other agents may offer additional benefits ${ }^{11,13}$. Our findings demonstrate that administration of rituximab along with cyclophosphamide and steroids in patients with AlHA and underlying lymphoproliferative disorders may result in an optimal approach with an effective and durable inmmunosuppresive response without significant toxicity. Accordingly, it has been reported that rituximab, cyclophosphamide and steroids combination is very effective in treating immune cytopenic diseases (ITP, AlHA) associated with chronic linfoproliferative syndromes ${ }^{10,13}$.

The optimal rituximab dosing scheme in autoimmune diseases has not been established. In all reported series, the typical dosing regimen for refractory AIHA patients is a weekly dose of $375 \mathrm{mg} / \mathrm{m}^{2}$ of rituximab for 2 or 4 weeks ${ }^{2}$. In our series, most of patients received 4 doses of rituximab and 7 patients received between 1-6 doses of rituximab without clear differences in their responses.

Rituximab may probably be less effective in patients previously treated for long periods of time with different immunosuppressive drugs, besides causing damage in these heavily treated patients ${ }^{26}$. Nevertheless, our results indicate that rituximab could induce excellent responses even in heavily treated refractory AlHA patients and accordingly, this agent could be a fine option as a second line of therapy before splenectomy. 
Treatment toxicity was minimal in our study, and no infectious processes were reported. None of the patients developed neutropenia. In similar studies, the doses of rituximab used in the present analysis were also well tolerated ${ }^{27,28}$. Rituximab has demonstrated a well-established safety profile through its use in hemato-oncological indications for over 10 years $^{29}$ and it has been used for treatment of $A D$ in children, adolescents and old patients with a good tolerability ${ }^{11,28,30}$. However, minor treatable infections have been common in patients with B cell depletion, whereas severe infections occurred in a small minority $^{29}$. In a recent review $^{31}$ regarding the use the rituximab in $A D$, an incidence of serious infections of $12.5 \%$ was reported. In AlHA patients (5 studies, 76 patients), 5 serious infections were reported and 2 patients died of septicemia. The frequency of infections in patients with $A D$ treated with rituximab depends on several factors. Therefore, concomitant immunosuppresive therapies ( $T$ cell immunosuppressive agents), which also enhance or contribute to susceptibility to infection, age of patient and the presence of other medical problems have been involved. Rituximab has been associated with viral infections including hepatitis B, cytomegalovirus, herpes simplex virus, varicella zoster virus, West Nile virus and JC virus ${ }^{32}$. Recently, 57 patients with progressive multifocal leucoencephalopathy (PML) after rituximab treatment have been reported ${ }^{33}$. Fifty-two patients suffered from LD and 5 patients had AD. One patient with AIHA developed PML after treatment with corticosteroids and rituximab.

In conclusion, rituximab induces a clinical effect not seen with other therapies in AlHA patients refractory to several treatments with little or tolerable associated toxicity. Therefore, this therapy should be considered as a potential early 
treatment that may achieve a rapid and MR in this subset of patients. Achievement of CR must be the therapeutic objective of rituximab in these patients. Furthermore, we could consider a negative Coombs test after treatment as a predictor of maintenance of response. The clinical effect of rituximab and its minimal toxicity could reduce the need for immunosuppressive agents avoiding their cytotoxic effects. Due to the excellent responses associated to rituximab in combination with cyclophosphamide and steroids, this therapy should be considered as a potential treatment of AlHA associated to LD. Additional large studies are required to answer key questions regarding dosage of rituximab, its optimal position in the therapeutic armamentarium of AlHA and the role of retreatment and combination therapy in order to enhance its therapeutical effect and duration of response. 
Acknowledgements: Member of the Spanish Study Group on the use of rituximab in refractory AIHA: Subirá, Hospital Sant Joan de Deu; Miguel Canales; Hospital Universitario La Paz; Madrid; Eva González, ICO; José Nieto, Hospital Morales Meseguer, Murcia; Magdalena Herrera, Hospital Nuestra Señora de la Candelaria, Tenerife; Manuel Andrés García, Hospital Perpetuo Socorro, Badajoz; Rodríguez Salazar, Hospital Universitario de Canarias; R. Hernández (Carmen Menchaca), Hospital Txagorritxu; Jose Ignacio Olalla, Hospital Sierrallana; Luis Palomera; Hospital Clínico Lozano Blesa. 


\section{Figure legends}

Figure 1. Response to rituximab therapy. 22 patients achieved CR (61\%) and 6 patients obtained PR (16\%). The response was maintained (MR) in all patients that achieved CR, with a median duration of 19 months.

Figure 2. Timing of response to rituximab therapy. Most of the responses took place between the first and third week, (18 patients; $82 \%$ of the responders). Only five patients responded between 6-8 weeks.

Figure 3. Evolution of hemoglobin levels. Median of hemoglobin level prior to treatment was $7 \mathrm{~g} / \mathrm{dL}$, and at maximum response, median hemoglobin level was 13 $\mathrm{g} / \mathrm{dL}$. There was a significant increase of hemoglobin levels since pre-treatment conditions $(p<0.001)$.

Figure 4. Kaplan-Meier analysis of MR. The estimated mean of MR along the study period was 65.9 months (95\% confidence interval [Cl]: 52.9-78.9).

Figure 5. Relation between Coombs test and MR. 15 out of 18 patients with MR reached a negative result from Coomb's test, and the number of these patients was significantly higher in relation to those that reached MR but whose result from Coomb's test was positive $(p<0.001)$. CT indicates Coombs test. 


\section{References}

1 Gehrs, B.C, Friedberg, R.C. Autoimmune hemolytic anemia. Am Journal of Hematology 2002; 69:258-271.

2 King KE, Ness PM. Treatment of autoimmune haemolytic anemia. Semin Hematol 2005; 42:131-6.

${ }^{3}$ Petz, L.D. Treatment of autoimmune hemolytic anemias. Current Opinion in Hematology, 2001; 8:411-416.

4 Garvey B. Rituximab in the treatment of autoimmune haematological disorders. Br J Haematol. 2008; 141(2):149-69.

5 Semple, J.W, Freedman, J. Autoimmune pathogenesis and autoimmune hemolytic anemia. Seminars in Hematology, 2005; 42:122- 130.

${ }^{6}$ Robak T. Monoclonal antibodies in the treatment of autoimmune cytopenias. Eur J Haematol 2004; 72:79-88.

${ }^{7}$ Berentsen, S., Ulvestad, E., Gjertsen, B.T., Hjorth-Hansen, H., Langholm, R., Knutsen, $\mathrm{H}$. et al. Rituximab for primary chronic cold agglutinin disease: a prospective study of 37 courses of therapy in 27 patients. Blood 2004; 103: 2925-2928.

${ }^{8}$ Peñalver FJ, Jiménez-Yuste V, Almagro M, Alvarez-Larrán A, Rodríguez L, et al.; Multi-institutional Retrospective Spanish Study Group on the Use of Rituximab in Refractory ITP. Rituximab in the management of chronic immune thrombocytopenic purpura: an effective and safe therapeutic alternative in refractory patients. Ann Hematol. 2006 Jun; 85(6):400-6.

9 Berentsen S. Rituximab for the treatment of autoimmune cytopenias. Haematologica 2007, 92:1589-96.

10 Ramanathan S, Koutts J, Hertzberg MS. Two cases of refractory warm autoimmune hemolytic anemia treated with rituximab. Am J Hematol 2005; 78: 123-6.

11 Zecca M, Nobili B, Ramenghi U, Perotta S, Amendola G, Rosito P et al. Rituximab for the treatment of refractory autoimmune hemolytic anemia in children. Blood 2003; 101:3857-3861.

12 Stasi R, Pagano A, Stipa E, Amadori S. Rituximab chimeric anti-CD20 monoclonal antibody treatment for adults with chronic idiopathic thrombocytopenic purpura. Blood 2001; 98:952-957.

${ }^{13}$ Zaja F, lacona I, Masolini P, Russo D, Sperotto A, Prosdocimo S. et al. B-cell depletion with rituximab as treatment for immune hemolytic anemia and thrombocytopenia. Haematologica 2002; 87:189-195. 
${ }^{14}$ Bussone G, Ribeiro E, Dechartres A, Viallard JF, Bonnotte B, et al. Efficacy and safety of rituximab in adults' warm antibody autoimmune haemolytic anemia: retrospective analysis of 27 cases. Am J Hematol. 2009; 84(3):153-7.

${ }^{15}$ Cooper N, Stasi R, Cunningham-Rundles S, Feuerstein MA, Leonard JP, Amadori S, Bussel JB. The efficacy and safety of B-cell depletion with antiCD20 monoclonal antibody in adults with chronic immune thrombocytopenic purpura. Br J Haematol 2004; 125:232-239.

${ }^{16}$ Zaja, F., Vianelli, N., Sperotto, A., Patriarca, F., Tani, M., Marin, L. et al. AntiCD20 therapy for chronic lymphocytic leukemia-associated autoimmune diseases. Leukemia \& Lymphoma 2003; 44: 1951-1955.

${ }^{17}$ Kajouri K, Vesely SK, Terrell DR, George JN. Splenectomy for adults patients with idiopathic thrombocytopenic purpura: a systematic review to assess longterm platelet count response, prediction of response, and surgical complications. Blood 2004; 104:2623-2634.

18 Kakaiya R. New Therapy with Anti-CD20 Antibody /rituximab) for Autoimmune Hemolytic anemia. Blood Therapies in Medicine 2003; 3: 91-96.

${ }^{19}$ Gupta, V. Tilak and Bhatia B.D. Immune Thrombocytopenic Purpura. Indian Journal of Pediatrics 2008: 75.

${ }^{20}$ Gertz MA. Management of cold haemolytic syndrome. Br J Haematol 2007; 138: 422-9.

${ }^{21}$ Shanafelt TD, Madume HL, Wolf RC, Tefferi A. Rituximab for immune cytopenia in adults: idiopathic thrombocytopenic purpura, autoimmune haemolytic anemia, and Evans syndrome. Mayo Clin Proc 2003; 78:1340-46.

${ }^{22}$ Berentsen, S., Ulvestad, E., Gjertsen, B.T., Hjorth-Hansen, H., Langholm, R., Knutsen, $\mathrm{H}$. et al. Rituximab for primary chronic cold agglutinin disease: a prospective study of 37 courses of therapy in 27 patients. Blood 2004; 103: 2925-2928.

${ }^{23}$ Schöllkopf, C., Kjeldsen L., Bjerrum O.W., Mourits-Andersen H.T., Nielsen J.L., Christensen B.E. et al. Rituximab in chronic cold agglutinin disease: a prospective study of 20 patients. Leukemia \& Lymphoma 2006; 47: 253-260.

${ }^{24}$ Shan D, Ledbetter JA, Press OW. Signaling events involved in anti-CD20induced apoptosis of malignant human B cells. Cancer Immunol Immunother 2000; 48:673-83.

25 Alas S, Emmanouilides C, Bonavida B. Inhibition of interleukin 10 by rituximab results in down-regulation of bcl-2 and sensitization of B-cell nonHodgkin's lymphoma to apoptosis. Clin Cancer Res 2001; 7:709-23.

26 Virgolini L, Marzocchi V Rituximab in autoimmune diseases. Biomed Pharmacother 2004; 58:299-309. 
${ }^{27}$ Schwartz J, Leber MD, Gillis S, Giunta A, Eldor A, Bussel JB. Long-term follow-up after splenectomy performed for immune thrombocytopenic purpura (ITP). Am J Hematol 2003; 72:94-98.

${ }^{28}$ Rao A, Kelly M, Musselman M, Ramadas J, Wilson D, Grossman W, Shenoy S. Safety, efficacy, and immune reconstitution after rituximab therapy in pediatric patients with chronic or refractory hematologic autoimmune cytopenias. Pediatr Blood Cancer. 2008; 50: 822-5.

${ }^{29}$ Kimby, E. Tolerability and safety of rituximab (MabThera). Cancer Treatment Reviews 2005; 31, 456-473.

30 Bengston KL, Skinner MA, Ware RE. Successful use of anti-CD20 (Rituximab) in severe, life-threatening childhood immune thrombocytopenic purpura. J Pediatr 2003; 143:670-673.

${ }^{31}$ Gürcan HM, Keskin DB, Stern JN, Nitzberg MA, Shekhani H, Ahmed AR. A review of the current use of rituximab in autoimmune diseases. Int Immunopharmacol. 2009; 9(1):10-25.

${ }^{32}$ Aksoy S, Harputluoglu H, Kilickap S, Dede DS, Dizdar O, Altundag K, Barista I. Rituximab-related viral infections in lymphoma patients. Leuk Lymphoma. 2007; 48(7):1307-12.

33 Carson KR, Evens AM, Richey EA, et al. Progressive multifocal leukoencephalopathy following rituximab therapy in HIV negative patients: a report of 57 cases from the Research on Adverse Drug Event and Reports (RADAR) project. Blood 2009; 113: 4834-40. 


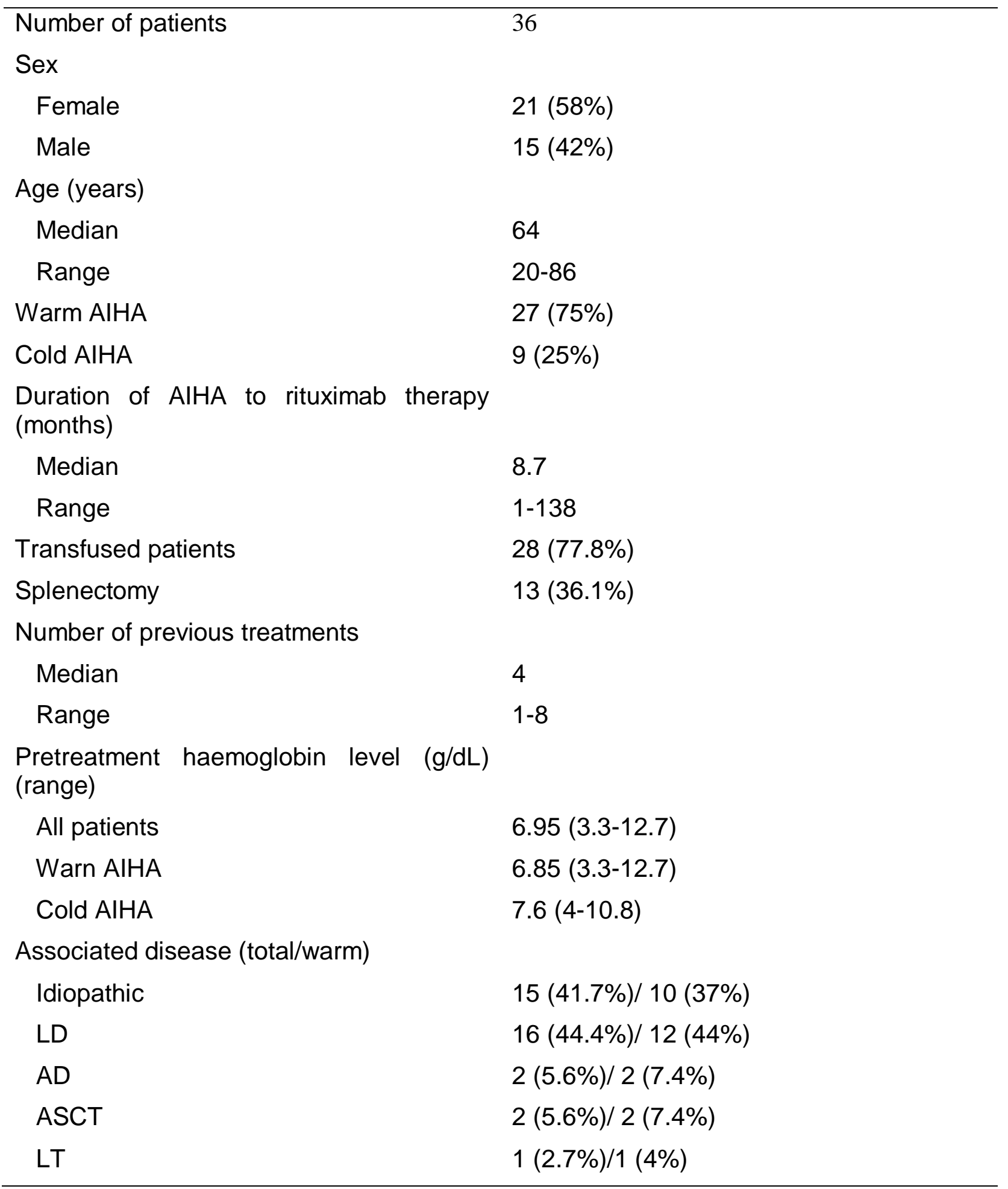

Table 1. Patients' clinical characteristics. The present table exhibits basal patients' characteristics. LD indicates lymphoproliferative disorders; AD, autoimmune disease; ASCT, allogenic stem cell transplant; LT, liver transplant. 
Click here to download high resolution image

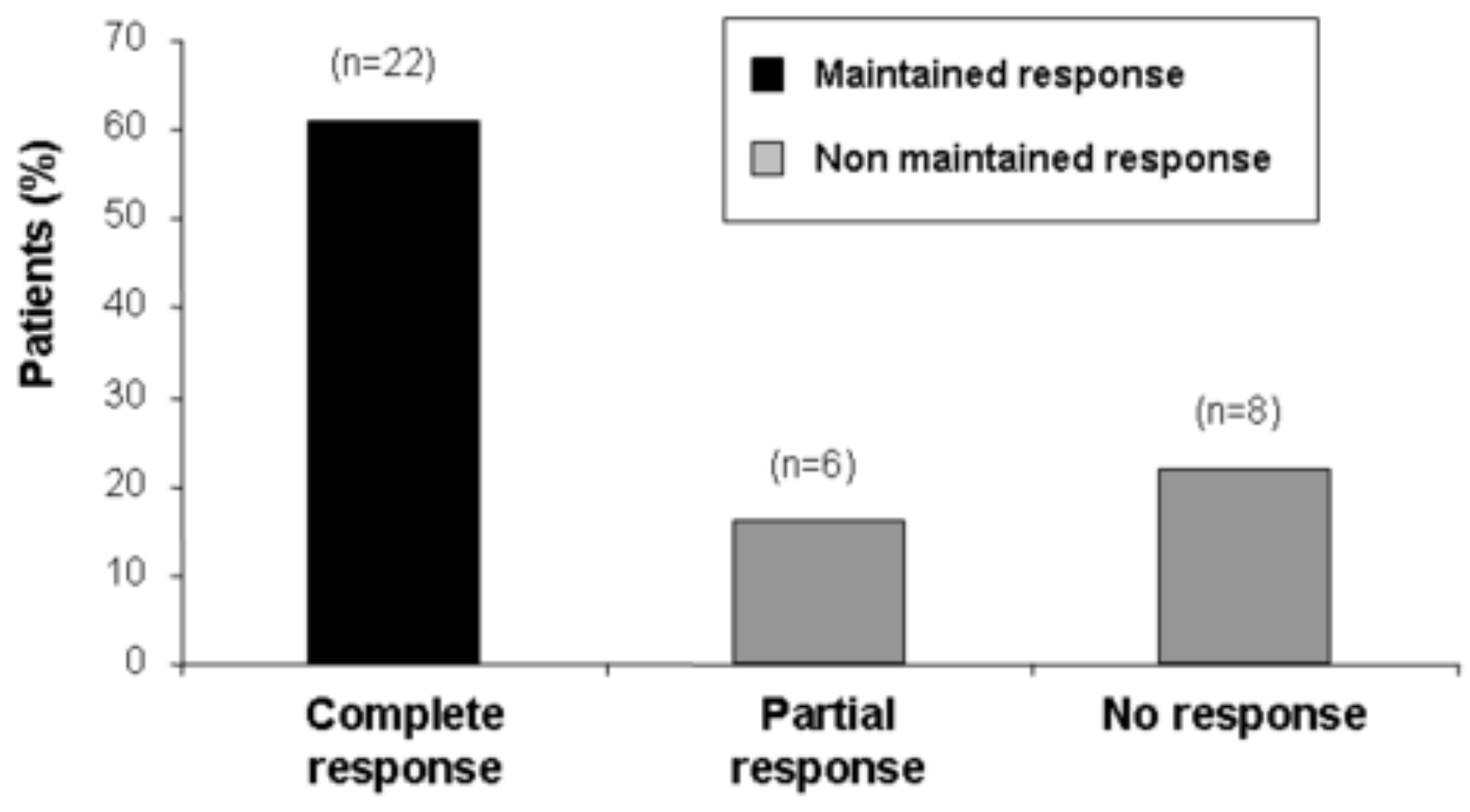


Click here to download high resolution image

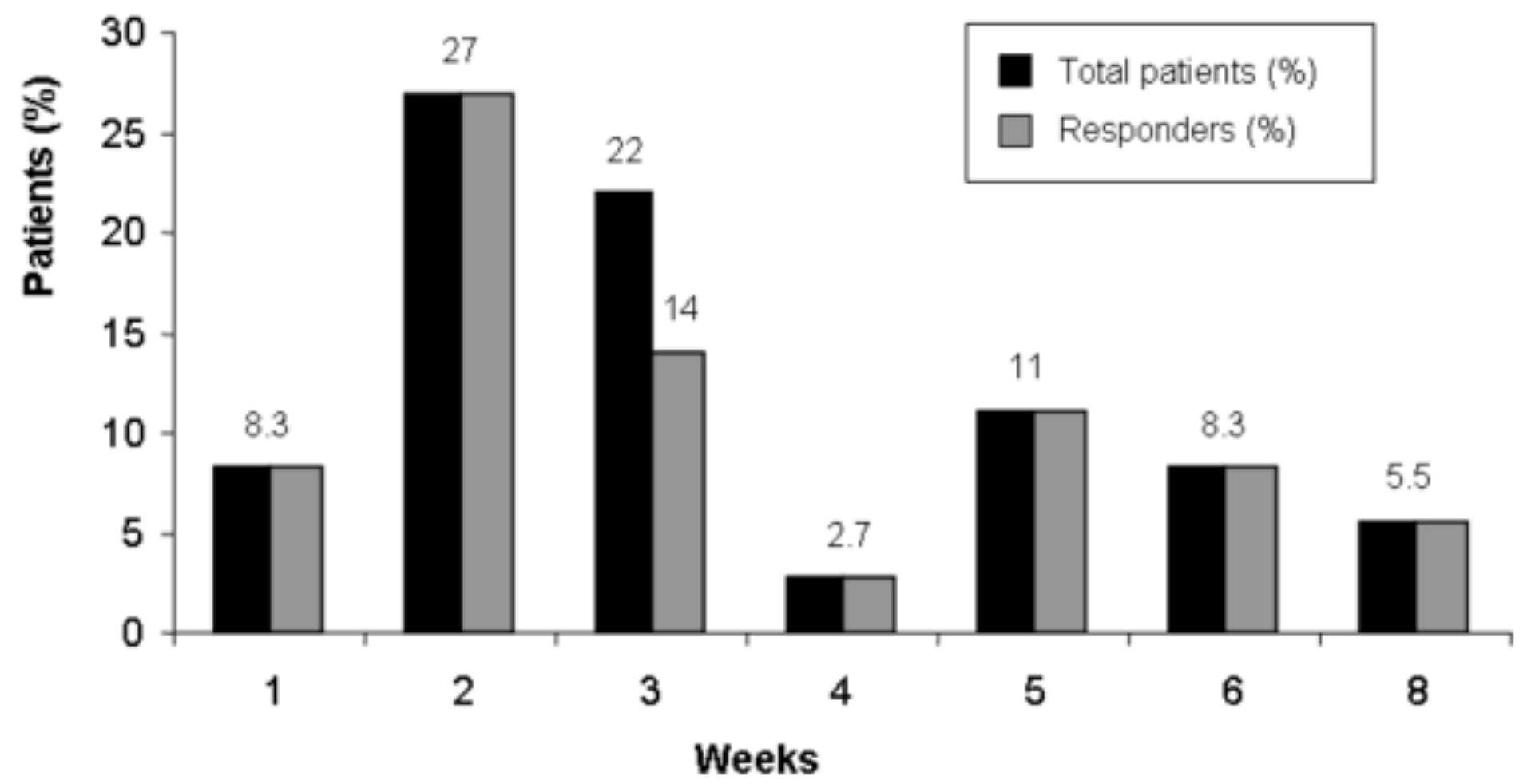




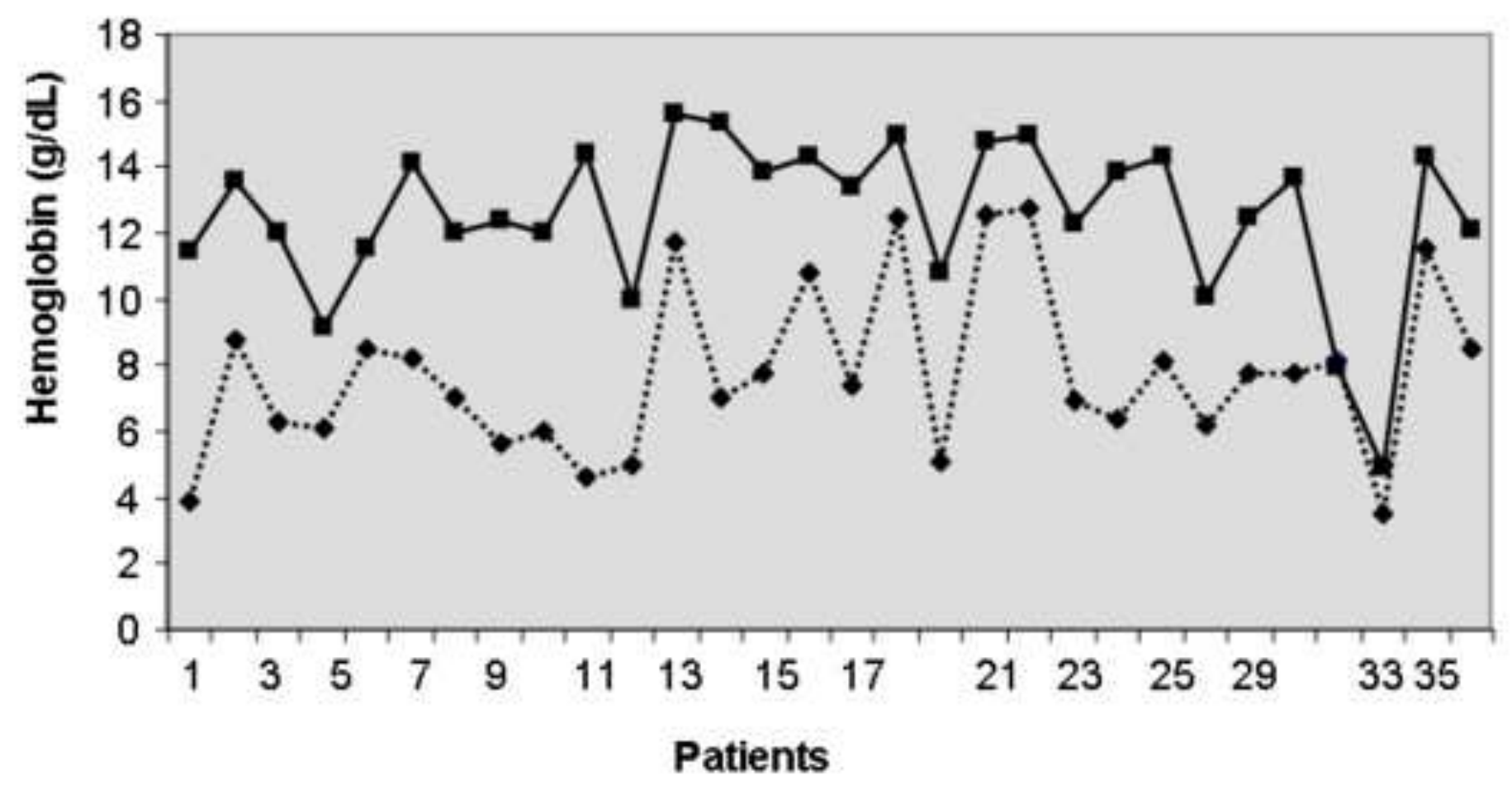

- Pre-treatment hemoglobin levels

......... Maximum response hemoglobin levels 
Click here to download high resolution image

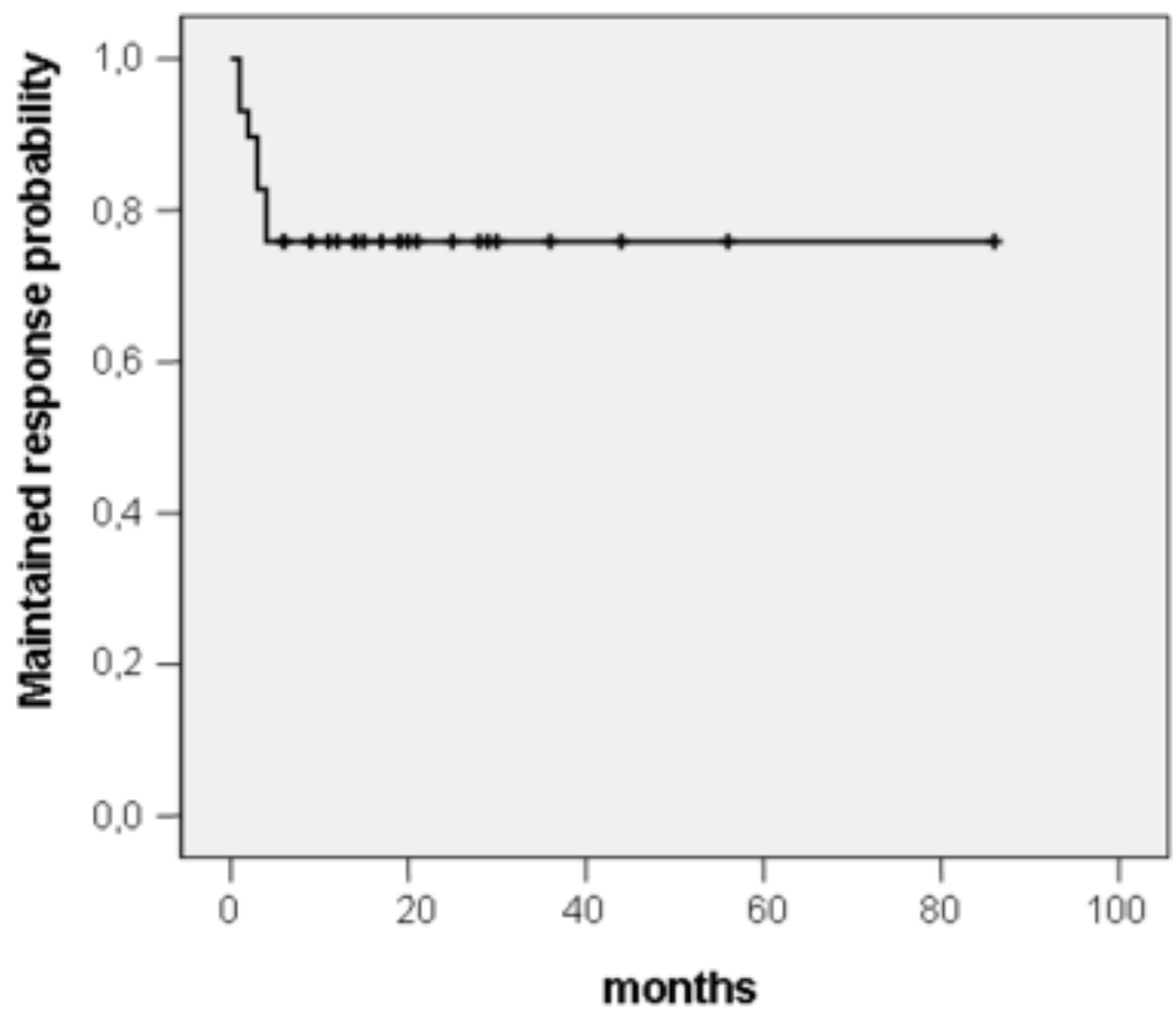




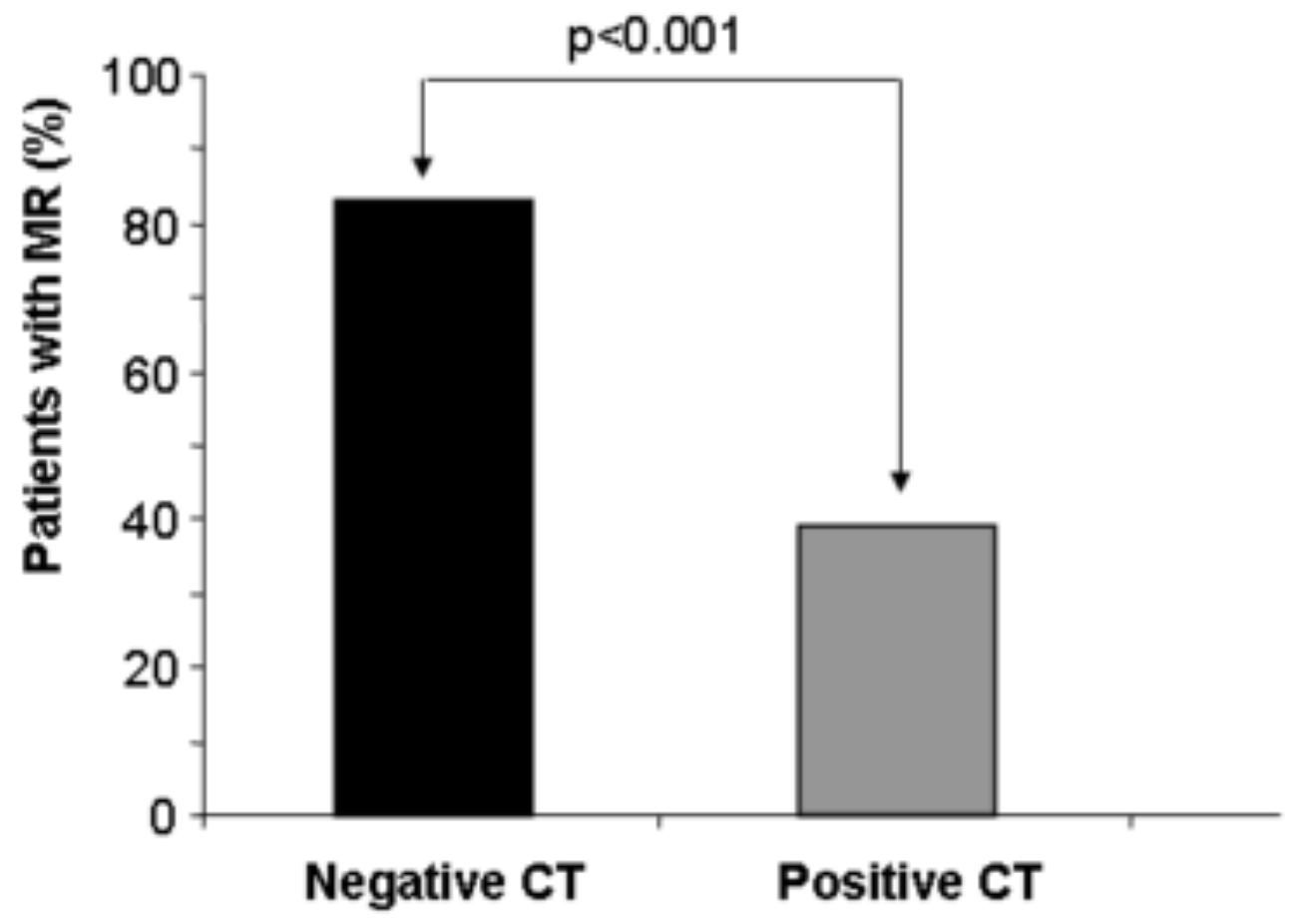


Manuscript ID: AOHE-D-09-00144

RITUXIMAB IS AN EFFECTIVE AND SAFE THERAPEUTIC ALTERNATIVE IN ADULTS WITH REFRACTORY AND SEVERE AUTOIMMUNE HEMOLYTIC ANEMIA

\section{Reviewers' comments:}

Reviewer \#2: All of my concerns have been addressed by the authors.

The paper needs editing by a native English speaker.

Discussion page 17:

- Rows 2 to 12 . This paragraph does not sound well

- Row 17: infections

- Row 19: autoimmune

The manuscript has been carefully revised in order to improve the language and style and some grammatical errors have been correct as advised by the reviewers.

Discussion page 17:

- Rows 2 to 12. This paragraph does not sound well

This paragraph has been re-written in order to clarify the content.

- Row 17: infections

- Row 19: autoimmune

We have corrected the grammatical errors regarding these words in the Discussion section. 